\title{
BMJ Open Intra-articular corticosteroids for treatment of temporomandibular joint internal disorders: protocol for systematic review and network meta- analysis
}

Daniela Torres, ${ }^{1,2}$ Carlos Zaror (D) , ${ }^{3,4}$ Verónica Iturriaga, ${ }^{5}$ Aurelio Tobias ${ }^{6}$

To cite: Torres D, Zaror C, Iturriaga $\mathrm{V}$, et al. Intra-articular corticosteroids for treatment of temporomandibular joint internal disorders: protocol for systematic review and network meta-analysis. BMJ Open 2020;10:e034327. doi:10.1136/ bmjopen-2019-034327

- Prepublication history and additional material for this paper are available online. To view these files, please visit the journal online (http://dx.doi org/10.1136/bmjopen-2019034327).

Received 15 September 2019 Revised 09 March 2020 Accepted 30 April 2020
Check for updates

(c) Author(s) (or their employer(s)) 2020. Re-use permitted under CC BY-NC. No commercial re-use. See rights and permissions. Published by BMJ.

For numbered affiliations see end of article.

Correspondence to

Prof. Carlos Zaror;

carlos.zaror@ufrontera.cl

\section{ABSTRACT}

Introduction Internal temporomandibular joint (TMJ) disorders are present in approximately $80 \%$ of patients with symptomatic temporomandibular disorders. Among the minimally invasive therapies, we find the intraarticular infiltration of substances, such as corticosteroids, hyaluronic acid or platelet-rich plasma accompanied or not by an arthrocentesis. There are several studies on minimally invasive therapy for internal TMJ disorders; however, none compares the effectiveness of the different intra-articular corticosteroids to each other.

The purpose of this study is to evaluate the effectiveness of the different intra-articular corticosteroids for the treatment of internal disorders of the TMJ and compare them to each other or to other minimally invasive therapies.

Methods and analysis A systematic search will be carried out up to December 2019 in the electronic databases: Medline, Cochrane Library, EMBASE, SCOPUS and LILACS.

Randomised clinical trials evaluating patients with internal disorders of the TMJ, with intra-articular corticosteroid therapy and comparing these to each other and/or to other minimally invasive therapy will be included. The main outcomes will be pain and range of motion measured through validated scales.

Two review authors will independently screen search results, extract data from included studies and assess the risk of bias in those studies using the Revised Cochrane Risk of Bias Tool (RoB 2.0). In the case of any discrepancy and failure to reach consensus, this will be resolved by a third reviewer.

A network meta-analysis will be conducted based on direct comparisons to generate indirect comparisons of the different treatments. Data will be combined in a metaanalysis using a random effects model.

The principles of the Grading of Recommendations Assessment, Development and Evaluation (GRADE) system will be used to assess the overall quality of the body of evidence associated with the main results.

Ethics and dissemination This protocol will not require ethical approval. The results of this review will be disseminated through peer-reviewed publications. Trial registration number CRD42019129014.
Strengths and limitations of this study

- This study will be the first network meta-analysis to evaluate the effectiveness of the different intraarticular corticosteroids used for internal temporomandibular joint (TMJ) disorders.

- It will evaluate which minimally invasive treatment is better in relation to pain management and ranges of motion in the internal disorders of the TMJ.

- The results of this systematic review will provide evidence to fill the gap in the knowledge about the most effective intra-articular corticosteroid for management of internal disorders of the TMJ.

- We will use the GRADE working group's approach to evaluate the quality of evidence.

- The main limitation may be the lack of available clinical trials that directly compare the different intraarticular corticosteroids.

\section{INTRODUCTION}

Temporomandibular disorders (TMD) are a wide group of pathologies that affect the temporomandibular joint (TMJ) and its adjoining structures. ${ }^{1}$ They are characterised mainly by pain, decreased range of motion and joint noise. ${ }^{2}$ Disorders of the TMJ include extra and intracapsular disorders. Extracapsular disorders affect the structures surrounding the TMJ, while intracapsular or internal disorders of the TMJ affect the structures inside it. ${ }^{3}$ Internal TMJ disorders are present in approximately $80 \%$ of patients with symptomatic TMD,${ }^{4}$ where inflammatory and nociceptive mediators are present in the synovial fluid of these joints. ${ }^{5}$

The treatment of internal TMJ disorders can range from conservative, minimally invasive and invasive therapies. Among the minimally invasive therapies, we find the intra-articular infiltration of substances, such as corticosteroids, hyaluronic acid (HA) and 
platelet-rich plasma, accompanied or not by an arthrocentesis. ${ }^{67}$

Corticoids or corticosteroids are a variety of hormones from the group of steroids, produced by the adrenal glands located in the upper part of the kidneys. ${ }^{89}$ The effects of corticosteroids can be divided into glucocorticoid for its regulating activity of carbohydrate metabolism, and mineralocorticoid for its regulating activity of electrolyte balance.

Corticosteroids are involved in a variety of physiological mechanisms, including their anti-inflammatory effect, their effect on the immune system, the metabolism of carbohydrates, protein catabolism, plasma electrolyte levels and others. ${ }^{10}$ They can be synthesised artificially and have therapeutic anti-inflammatory applications, preventing the release of arachidonic acid by inhibiting phospholipase A2, which in turn prevents the formation of proinflammatory cytokines and harmful mediators. ${ }^{11}$ Corticosteroids have been used therapeutically in the inflammation of different joints, showing positive results in small joints, such as the trapeziometacarpal. ${ }^{12}$

Corticosteroids are one of the tools used in the management of TMJ internal disorders, generating the best anti-inflammatory effect due to the inhibition of the production and secretion of proinflammatory cytokines such as interleukins, tumour necrosis factor alpha, interferon gamma and factor stimulating granulocytic and macrophage colonies by direct interference on cascades and genomic mechanisms. They also inhibit the accumulation of macrophages and neutrophils in inflammatory foci because they repress the expression of endothelial adhesion molecules and the synthesis of the plasminogen activator. ${ }^{9}$ There is evidence that they are effective in controlling pain and functional capacity, with few side effects. $^{13}$

Today, there are several studies on minimally invasive therapy for internal TMJ disorders; however, none compares the effectiveness of the different intra-articular corticosteroids, thus making it difficult to determine which is the most effective in relation to pain management and range of motion. ${ }^{14}$

When evaluating the effects of different corticosteroids on the articular cartilage of various joints, the most commonly used in the treatment of pain are hydrocortisone, methylprednisolone, dexamethasone, betamethasone, prednisolone and triamcinolone. ${ }^{15}$

A network meta-analysis will allow direct and indirect comparisons, which can be used not only to strengthen the inferences about the effectiveness of intra-articular corticosteroids, but also to determine the effectiveness of other minimally invasive therapies and to compare them, such as the application of HA, arthrocentesis or plateletrich plasma.

The objectives of this systematic review and the network meta-analysis are: (1) to determine the most effective intra-articular corticosteroid in relation to pain management and range of motion in the internal disorders of the TMJ; (2) to compare the effectiveness of intra-articular corticosteroid infiltrations against other minimally invasive therapies in relation to pain and range of motion in internal TMJ disorders; (3) to determine the adverse events of intra-articular infiltrations of corticosteroids in the TMJ; (4) to determine the improvement in the quality of life of patients treated with intra-articular corticosteroids and (5) to determine the cost-effectiveness of intraarticular corticosteroid therapy.

The results of this study will provide important information for decision-making in specialist clinical practice.

\section{METHODS AND ANALYSIS}

This protocol was registered in PROSPERO and prepared according to the reporting guide provided in the Preferred Report Elements for Systematic Reviews and Meta-analysis Protocols (PRISMA-P). ${ }^{16}$

\section{Patient and public involvement}

Patients and or public were not be involved in this study, either in planning or the design of the study. Patients were not invited to comment on the study design and were not consulted to develop patient-relevant outcomes or interpret the results. Patients were not invited to contribute to the writing or editing of this document for readability or accuracy.

\section{Eligibility criteria}

\section{Study types}

Randomised controlled clinical trials in English, Spanish, French, Portuguese and German will be included.

\section{Types of participants}

We will include patients with internal disorders of the TMJ, such as disc displacements or osteoarthritis, and those without systemic diseases. That is, any disease that affects other parts of the body, or even the entire body and which may interfere with the outcome of the intervention, such as rheumatic disorders and metabolic diseases among others.

\section{Type of interventions}

The intervention will be through the use of $0.5-1 \mathrm{~mL}$ of intra-articular corticosteroids in the treatment of internal TMJ disorders, such as triamcinolone, betamethasone, dexamethasone, hydrocortisone, methylprednisolone.

The different corticosteroids will be compared with each other or to another minimally invasive therapy for internal TMJ disorders, such as HA, autologous plateletrich plasma or arthrocentesis.

\section{Outcome measures}

These outcomes are likely to be the most clinically important and commonly reported events in the studies of relevance.

\section{Primary outcomes}

- The effectiveness of intra-articular corticosteroids for the treatment of internal disorders of the TMJ, 
measured through validated pain scale, such as the visual analogue scale and range of motion. The range of motion will be evaluated through the measurement of the oral opening in millimetres. All outcomes will be measured at 1, 3, 6 and 12 months, due to the clinical significance of their anti-inflammatory action during this time period.

\section{Secondary outcomes}

- Adverse events of intra-articular infiltrations of corticosteroids in the TMJ, such as inflammation, pain, temporary swelling, haemorrhage or limitation of mouth opening. These will be reported separately and as a total number of adverse effects.

- Quality of life of patients treated with corticosteroids, measured using a validated scale, such as Oral Health Impact Profile (OHIP), European Quality of Life-5 Dimensions (EQ-5D), Short Form-36 Health Survey (SF-36) and Short Form-12 Heath Survey (SF-12).

- Cost-effectiveness of intra-articular corticosteroid therapy in internal TMJ disorders, measured by adjusted quality of life years or disability-adjusted life years or another appropriate measure.

\section{Search strategy}

A systematic search will be carried out up to December 2019 in the following databases: Medline, Cochrane Central Register of Controlled Trials, EMBASE, SCOPUS and LILACS. The details of the search strategy used in Medline (PubMed) is listed in online supplementary file.

The search strategy in Medline will be adapted for the other databases according to the requirements of each. Grey literature will be obtained through searches in OpenGray database (www.opengrey.eu) and trial registers such as US National Institutes of Health Ongoing Trials Register ClinicalTrials.gov (clinicaltrials.gov) and WHO International Clinical Trials Registry Platform (apps. who.int/trialsearch) to identify ongoing clinical trials and conference proceedings. We will also examine the reference lists of the included articles and those previous systematic reviews that were checked to identify other possible studies that could be included.

\section{Data management}

All references identified will be extracted to an EndNote V.X9 database to facilitate their management and delete duplicates. Selection process and data extraction will be carried out using the Covidence software (http://www. covidence.org).

Titles and/or abstracts of studies retrieved using the search strategy and those from additional sources will be screened independently by two review authors to identify studies that potentially meet the inclusion criteria. We will obtain full copies of all relevant and potentially relevant studies, those appearing to meet the inclusion criteria, and those for which there are insufficient data in the title and abstract to make a clear decision. Any disagreement between the two review authors over the eligibility of particular studies will be resolved through discussion with a third reviewer. The reasons for excluding judgements will be recorded.

A standardised, pre-piloted form will be used to extract data from the included studies regarding individual trial characteristics. Extracted information will include: study design/setting, study population and participant demographics, baseline characteristics, details of the intervention and control conditions, outcome data of interest, follow-up times. Two review authors will extract data independently, discrepancies will be identified and resolved through discussion (with a third author if necessary). Authors will be contacted to identify unpublished or ongoing clinical trials and to clarify data as required.

\section{Risk of bias in individual studies}

Two reviewers will independently assess the risk of bias in the studies included in accordance with Revised Cochrane Risk of Bias Tool (RoB 2.0).${ }^{17}$ We will examine the suitability of the method used based on five domains: bias derived from the randomisation process; bias due to deviations from planned interventions; bias due to lack of results data; bias in the measurement of the result and bias in the selection of the reported result. A series of signalling questions will be included for each domain that aim to provide a structured approach to obtain relevant information on bias risk assessment. For each domain the possible risk of bias judgements will be: low risk of bias, some concerns and high risk of bias. We will also present a summary of 'risk of bias' graphically.

\section{Dealing with missing data}

The original authors of the study will be contacted whenever possible, to obtain the missing data and details of any outcomes that may have been measured but not reported. An intention-to-treat approach will be carried out for the primary analysis, where we will include all participants randomised to each group, regardless of whether they received the allocated intervention or not. We will not use any other statistical methods or perform any further imputation to account for missing data.

\section{Statistical analysis}

For continuous outcomes, effect measures such as mean difference or standardised mean difference (SMD) will be used. The scale of the available data will be primarily used to determine the choice of effect measure if studies report effect estimates with the same or similar scale Weighted Mean Difference (WMD) or when the outcome is measured using different scales (SMD) ${ }^{17}$ For binary outcomes, the relative risk will be used as the standard effect measure of association across studies.

We will carry out meta-analyses at pre-specified follow-up times based on available data. Initially, we will perform pairwise meta-analyses using a random-effects model for every treatment comparison with at least two studies. ${ }^{18}$ Next, we will generate and assess the network diagrams to determine if a network meta-analysis is feasible. We will 
perform the network meta-analysis within a frequentist framework using multivariate meta-analysis estimated by restricted maximum likelihood. All analyses will be done using the Stata statistical software, release 15 (StataCorp, College Station, Texas, USA). We will use the network suite of Stata commands designed for this purpose. ${ }^{19}$

If pooling is not appropriate, we plan to perform only descriptive analysis.

\section{Assessment of heterogeneity}

We will assess the clinical heterogeneity in the included studies by examining the similarity between the types of participants, interventions and outcomes. In pairwise meta-analyses, we will estimate the heterogeneity for each comparison. ${ }^{17}$ We will assess the heterogeneity statistically using the $\chi^{2}$ test, where a $p$ value $<0.1$ indicated statistically significant heterogeneity. Additionally, we will quantify heterogeneity using the $\mathrm{I}^{2}$ statistic, with values over $50 \%$ indicating considerable heterogeneity. ${ }^{17}$ In the network meta-analysis, we will assume a common estimate for the heterogeneity variance across all of the different comparisons.

\section{Assessment of transitivity}

In this context, we expect that the transitivity assumption holds assuming that the common treatment used to compare different corticosteroids indirectly is similar when it appears in different trials. The assumption of transitivity will be evaluated epidemiologically by comparing the clinical and methodological characteristics of sets of studies from the various treatment comparisons.

\section{Assessment of inconsistency}

To check the assumption of consistency in the entire network, we will use the 'design-by-treatment' model. ${ }^{20}$ This method accounts for different sources of inconsistency that can occur when studies with different designs (two-arm trials vs three-arm trials) give different results as well as disagreement between direct and indirect evidence. Moreover, we will use the node-splitting method as further exploration if any inconsistency is found. ${ }^{21}$ This method evaluates the consistency assumption in each closed loop of the network separately as the difference between direct and indirect estimates for a specific comparison in the loop (inconsistency factor). Then, the magnitude of the inconsistency factors and their 95\% CIs can be used to infer about the presence of inconsistency in each loop. We will assume a common heterogeneity estimate within each loop.

\section{Relative treatment ranking}

We will estimate the ranking probabilities for all treatments of being at each possible rank for each intervention, with its $95 \% \mathrm{CI}^{22}$ Next, we will estimate the cumulative probabilities for each treatment being at each possible rank and will determine a treatment hierarchy using the surface under the cumulative ranking curve and its $95 \% \mathrm{CIs}^{22}$

\section{Additional analysis}

We plan to carry out the following subgroup analyses according to the duration of follow-up (short duration ( $<6$ months) vs long duration ( $>6$ months) ) and quality of life questionnaires (health-related quality of life instruments vs oral health-related quality of life instruments) as a possible source of heterogeneity between studies. We will also conduct a sensitivity analysis for each outcome by excluding studies with an overall high risk of bias. In addition, we will perform a worst-best and best-worst case scenario sensitivity analysis to assess the effect of missing data in trials with a large number of patient loss (over $20 \%)$.

\section{Assessment of reporting biases}

We will assess evidence of publication bias if more than 10 studies are to be included in the synthesis by using Begg's rank correlation test and examined using funnel plots. ${ }^{23}$ If asymmetry is detected, causes other than publication bias will be explored, such as selective outcome reporting, poor methodological quality in smaller studies, true heterogeneity, artefactual and chance. In addition, a contour-enhanced funnel plots will be performed to differentiate if the asymmetry is due to reporting biases or due to factors other than non-reporting biases, for example, confounding factors such as differential study quality. ${ }^{24}$

\section{Reporting conclusions}

The systematic review will be conducted and reported in accordance with the PRISMA-Network Meta-Analysis (NMA) $)^{25}$ standards.

We will use the GRADE working group's approach ${ }^{26} 27$ for rating the certainty of the network meta-analysis effect estimates for all the comparisons and all outcomes. We will appraise certainty of the direct, indirect and network evidence sequentially (in this order), following these four steps to assess the quality of treatment effect estimates from NMA. We will (1) present direct and indirect treatment estimates for each comparison of the evidence network. The direct estimate of effect is provided by a head-to-head comparison (trials of A vs B), and the indirect estimate is provided by two or more head-to-head comparisons that share a common comparator (eg, we infer the effects of A vs B from trials of A vs $\mathrm{C}$ and trials of $\mathrm{B}$ vs C); (2) Rate the quality of each direct and indirect effect estimate; (3) Present the NMA estimate for each comparison of the evidence network and (4) Rate the quality of each NMA effect estimate. ${ }^{27}$

A 'summary of findings' table for each comparison and for the main outcomes that include the effect estimate and certainty judgements for each direct evidence, indirect evidence and the network meta-analysis will be created. Certainty of evidence will be assessed across the domains of risk of bias, consistency, directness, precision and publication bias. Depending on the quality of the evidence, it can be downgraded one or two levels for 
each aspect. We will categorise the quality of each body of evidence as high, moderate, low or very low.

\section{Ethics and dissemination}

Formal ethical approval is not required as no primary data will be collected. The results of this review will be disseminated through peer-reviewed publications.

\section{Author affiliations}

${ }^{1}$ Master Program in Dentistry, Faculty of Dentistry, Universidad de La Frontera,

Temuco, Chile

${ }^{2}$ Temporomandibular Disorder and Orofacial Pain Program, Sleep \& Pain Research Group, Faculty of Dentistry, Universidad de La Frontera, Temuco, Chile

${ }^{3}$ Department of Pediatric Dentistry and Orthodontics, Faculty of Dentistry, Universidad de La Frontera, Temuco, Chile

${ }^{4}$ Faculty of Dentistry, Universidad San Sebastian, Puerto Montt, Chile ${ }^{5}$ Department of Integral Adult Care Dentistry, Temporomandibular Disorder and Orofacial Pain Program, Sleep \& Pain Research Group, Faculty of Dentistry, Universidad de La Frontera, Temuco, Chile

${ }^{6}$ Institute of Metabolism and Systems Research, University of Birmingham, Birmingham, UK

Acknowledgements DT is a MSc candidate of Master of Dentistry, Faculty of Dentistry, Universidad de La Frontera, Temuco, Chile.

Contributors DT, VI and CZ contributed to the conception and design of the study. DT and CZ developed the search strategies. AT designed the data analysis. All authors drafted the article and made the final approval of the version to be published.

Funding This project was funded by a grant from the Universidad de La Frontera, DIUFRO No. DI20-0133; Master Program in Dentistry, Universidad de La Frontera; Temporomandibular Disorders and Orofacial Pain Program, Universidad de La Frontera.

Competing interests None declared.

Patient consent for publication Not required.

Provenance and peer review Not commissioned; externally peer reviewed.

Supplemental material This content has been supplied by the author(s). It has not been vetted by BMJ Publishing Group Limited (BMJ) and may not have been peer-reviewed. Any opinions or recommendations discussed are solely those of the author(s) and are not endorsed by BMJ. BMJ disclaims all liability and responsibility arising from any reliance placed on the content. Where the content includes any translated material, BMJ does not warrant the accuracy and reliability of the translations (including but not limited to local regulations, clinical guidelines, terminology, drug names and drug dosages), and is not responsible for any error and/or omissions arising from translation and adaptation or otherwise.

Open access This is an open access article distributed in accordance with the Creative Commons Attribution Non Commercial (CC BY-NC 4.0) license, which permits others to distribute, remix, adapt, build upon this work non-commercially, and license their derivative works on different terms, provided the original work is properly cited, appropriate credit is given, any changes made indicated, and the use is non-commercial. See: http://creativecommons.org/licenses/by-nc/4.0/.

ORCID iD

Carlos Zaror http://orcid.org/0000-0001-6942-6956

\section{REFERENCES}

1 Di Paolo C, D'Urso A, Papi P, et al. Temporomandibular disorders and headache: a retrospective analysis of 1198 patients. Pain Res Manag 2017;2017:1-8.

2 Okeson JP. Etiology and identification of functional disturbances in the masticatory system. in: Okeson JP, editor. management of temporomandibular disorders and occlusion. 6th edn. Barcelona: Elsevier, 2008: 285-331.

3 Okeson JP. The classification of orofacial pains. Oral Maxillofac Surg Clin North Am 2008;20:133-44.

4 Paesani D, Westesson PL, Hatala M, et al. Prevalence of temporomandibular joint internal derangement in patients with craniomandibular disorders. Am J Orthod Dentofacial Orthop 1992;101:41-7.

5 Shafer DM, Assael L, White LB, et al. Tumor necrosis factor-alpha as a biochemical marker of pain and outcome in temporomandibular joints with internal derangements. J Oral Maxillofac Surg 1994;52:786-91.

6 Dym H, Bowler D, Zeidan J. Pharmacologic treatment for temporomandibular disorders. Dent Clin North Am 2016;60:367-79.

7 Moldez MA, Camones VR, Ramos GE, et al. Effectiveness of intraarticular injections of sodium hyaluronate or corticosteroids for intracapsular temporomandibular disorders: a systematic review and meta-analysis. J Oral Facial Pain Headache 2018;32:53-66.

8 McKay LI, Cidlowski JA. Physiologic and pharmacologic effects of corticosteroids. 6th edn, 2003.

9 Gómez S, Gutiérrez AM, Valenzuela EL. Corticoids: 60 years later a pending subject. Revista Ciencias de la Salud 2007;5:58-69.

10 Scherer J, Rainsford KD, Kean CA, et al. Pharmacology of intraarticular triamcinolone. Inflammopharmacology 2014;22:201-17.

11 Jacobs JW, Bijlsma JW, Gs F, et al. Glucocorticoid therapy. In: Kelley's Textbook of Rheumatology. Philadelphia: Elsevier, 2013: 894-916.

12 Cormier G, Le Goff B, Denis A, et al. Corticosteroids injections versus corticosteroids with hyaluronic acid injections in rhizarthrosis: the randomised multicentre RHIZ'ART trial study protocol. BMJ Open 2019;9:e022553.

13 Vargas F, Medina M, Hermosa J, et al. Treatment of patients with osteoarthritis. Atención Primaria 2014;46:S39-61.

14 Manfredini D, Rancitelli D, Ferronato G, et al. Arthrocentesis with or without additional drugs in temporomandibular joint inflammatorydegenerative disease: comparison of six treatment protocols*. $J$ Oral Rehabil 2012;39:245-51.

15 Wernecke C, Braun HJ, Dragoo JL. The effect of intra-articular corticosteroids on articular cartilage. Orthop J Sports Med 2015;3:232596711558116:1-7.

16 Moher D, Liberati A, Tetzlaff J, et al. Preferred reporting items for systematic reviews and meta-analyses: the PRISMA statement. PLoS Med 2009;6:1000097.

17 Higgins JPT, Thomas J, Chandler J. Cochrane Handbook for systematic reviews of interventions. Version 6. Cochrane, 2019. www. training.cochrane.org/handbook

18 DerSimonian R, Laird N. Meta-Analysis in clinical trials. Control Clin Trials 1986;7:177-88.

19 White IR. Network meta-analysis. Stata J 2015;15:951-85.

20 Higgins JPT, Jackson D, Barrett JK, et al. Consistency and inconsistency in network meta-analysis: concepts and models for multi-arm studies. Res Synth Methods 2012;3:98-110.

21 Dias S, Welton NJ, Caldwell DM, et al. Checking consistency in mixed treatment comparison meta-analysis. Stat Med 2010;29:932-44.

22 Salanti G, Ades AE, loannidis JPA. Graphical methods and numerical summaries for presenting results from multipletreatment meta-analysis: an overview and tutorial. $J$ Clin Epidemiol 2011;64:163-71.

23 Egger M, Davey Smith G, Schneider M, et al. Bias in meta-analysis detected by a simple, graphical test. BMJ 1997;315:629-34.

24 Page MJ, Higgins J, Sterne J, et al. Assessing risk of bias due to missing results in a synthesis. In: Cochrane Handbook for systematic reviews of interventions. 2019. version 6.0. Cochrane, 2019. www. training.cochrane.org/handbook

25 Hutton B, Salanti G, Caldwell DM, et al. The PRISMA extension statement for reporting of systematic reviews incorporating network meta-analyses of health care interventions: checklist and explanations. Ann Intern Med 2015:162:777-84.

26 Brignardello-Petersen R, Bonner A, Alexander PE, et al. Advances in the GRADE approach to rate the certainty in estimates from a network meta-analysis. J Clin Epidemiol 2018;93:36-44.

27 Puhan MA, Schünemann HJ, Murad MH, et al. A grade Working group approach for rating the quality of treatment effect estimates from network meta-analysis. BMJ 2014;349:g5630. 